\title{
BEACH VOLUME CHANGE USING UAV PHOTOGRAMMETRY SONGJUNG BEACH, KOREA
}

\author{
C. I. Yoo ${ }^{\mathrm{a}, *}$, T. S. Oh \\ ${ }^{a}$ Research Center for Ocean Industrial Development(RCOID), Pukyong National University, Yongso-ro, Nam-Gu, Busan, Korea - \\ mc2002@daum.net \\ ${ }^{\mathrm{b}}$ Dept. of Ocean Engineering, Pukyong National University, Yongso-ro, Nam-Gu, Busan, Korea - dhdbtkd@ naver.com
}

Commission VIII, WG VIII/9

KEY WORDS: Coastal Topographic Change, Unmanned Aerial Vehicle (UAV), RTK-VRS, Beach Volume Change

\begin{abstract}
:
Natural beach is controlled by many factors related to wave and tidal forces, wind, sediment, and initial topography. For this reason, if numerous topographic data of beach is accurately collected, coastal erosion/acceleration is able to be assessed and clarified. Generally, however, many studies on coastal erosion have limitation to analyse the whole beach, carried out of partial area as like shoreline (horizontal 2D) and beach profile (vertical 2D) on account of limitation of numerical simulation. This is an important application for prevention of coastal erosion, and UAV photogrammetry is also used to 3D topographic data. This paper analyses the use of unmanned aerial vehicles (UAV) to 3D map and beach volume change. UAV (Quadcopter) equipped with a non-metric camera was used to acquire images in Songjung beach which is located south-east Korea peninsula. The dynamics of beach topography, its geometric properties and estimates of eroded and deposited sand volumes were determined by combining elevation data with quarterly RTK-VRS measurements. To explore the new possibilities for assessment of coastal change we have developed a methodology for 3D analysis of coastal topography evolution based on existing high resolution elevation data combined with low coast, UAV and on-ground RTK-VRS surveys. DSMs were obtained by stereo-matching using Agisoft Photoscan. Using GCPs the vertical accuracy of the DSMs was found to be $10 \mathrm{~cm}$ or better. The resulting datasets were integrated in a local coordinates and the method proved to be a very useful fool for the detection of areas where coastal erosion occurs and for the quantification of beach change. The value of such analysis is illustrated by applications to coastal of South Korea sites that face significant management challenges.
\end{abstract}

\section{INTRODUCTION}

\subsection{Research Background}

Coastal areas contain a number of different types of site of particular biological, geological, geomorphological, landscape or cultural heritage value, each with specific requirements for monitoring. In addition, Natural beach is controlled by many coastal factors in regard to wave and tidal forces, wind, river sediment. Coastal topography, therefore, are very complex and dynamic in the extremely condition as like typhoons. However, in the implementation of any new scheme, it is highly that some form of topographic survey would be required, followed by various and accurate monitoring. Beach profiling coupled with profile analysis to estimate sediment volume changes has been identified as an essential component of the planning and evaluation procedures for such schemes as beach recharge, barrage construction, groyne construction, beach nourishment and managed retreat (Anon, 1993). Sediment volume change measurement is more useful for validating computer models of sediment transport and topographical change. Actually, acquiring such data for lager areas at short time intervals (less one year) using traditional ground based surveys would be prohibitively time consuming and expensive. Therefore, monitoring of coastal change has until been 2 dimension, focusing on shoreline evolution supported by a limited set of shore perpendicular profiles. Neither 2-D shoreline nor profile mapping can fully capture the rapid coastal changes caused by natural processes. Accurate data and a better understanding of coastal topographic change can significantly improve the success rate of such coastal environmental projects. Over the last few years, various achievements and improvements of the UAV system has been done by numerous researchers from different disciplines. UAV systems receive high demands in business, research institutes and industries since UAV is one of the simple and cheapest equipment in image acquisition. Indeed, it can fly at low altitude and provides very high resolution image of the ground surface. UAV also promised a low-cost flight mission alternative to the classical manned aircraft (Marzolff and Poesen, 2009).

To explore the new possibilities for assessment of beach volume change we have applied coastal topography of Songjung beach based on UAV survey with high resolution, on-ground RTK-VRS surveys.

\subsection{Study Area}

Songjung Beach is located on the south-east coast of Korea (Figure 1). The beach is one of the most famous beaches and highly valued for relaxation, sports and leisure. The beach, extending for less than $1 \mathrm{~km}$ in the NE-SW direction, is characterized by the presence of equipped sandy beaches. Even 
though reduce of sediment by tourism-related pressures, establishment of piers affecting the normal alongshore sediment drift, almost all this area is stable. It was selected as a study area due to its stable topographic features, fixed boundary (concrete structure).
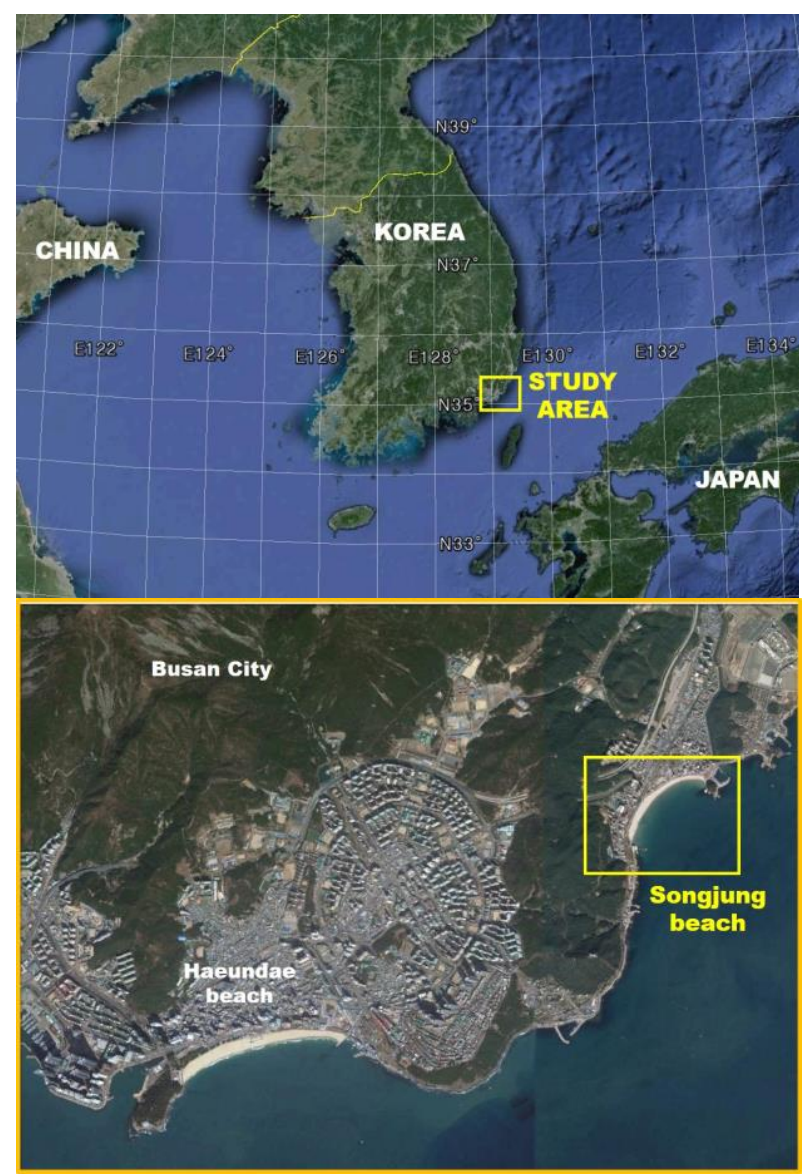

Figure 1. Location of study area (Songjung beach)

\subsection{Importance of beach volume}

Lidar \& UAS have become a huge benefit for those of us looking at coastal change. Quantifying change based on a beach profile every $\mathrm{km}$ or so has been replaced by tens of millions of points every $\mathrm{km}$, increasing the resolution of the change analysis and efficacy in doing kilometres of beach volume dramatically. It is important that when a municipality/province uses UAS to quantify how much sand they lost, so they can recoup some losses or plan on renourishment, they understand what the value represents. It can be easy to overlook some subtleties because, regardless of technique, the change will likely be a large number. In this paper the beach volume concept to deal with coastal erosion/deposition is further explored by identifying and analyzing the sediment volumes accumulated in large-scale and small-scale coastal areas at various study sites.

\section{DATA ACQUISITION}

\subsection{Sensor platform}

The unmanned aerial vehicle used for mapping was Inspire quad-copter drone (DJI, China) in Figure 1. The key specifications of the UAV are shown in Table 1, for the study area

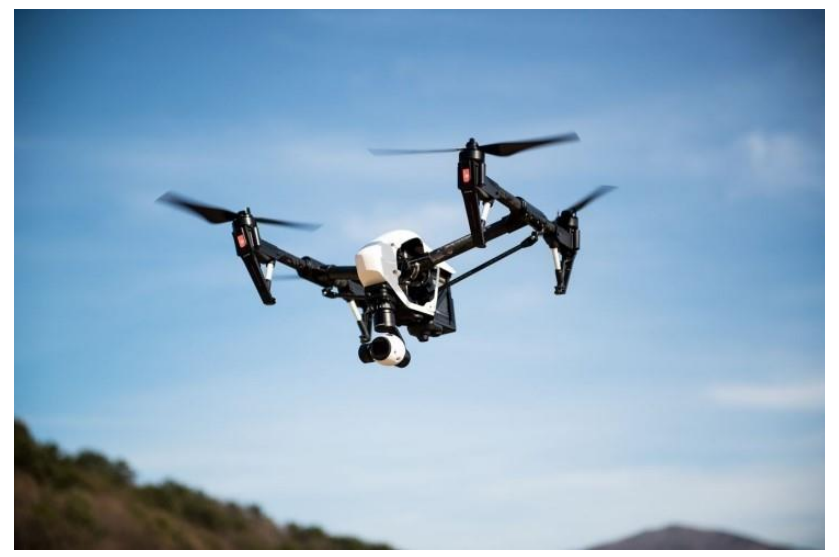

Figure 2.Inspire

\begin{tabular}{|l|c|}
\hline Manufacturer & Inspire DJI, China \\
\hline Type & Micro-drone Quadcopter \\
Engine Power & 4 Electric Brushless \\
Dimension and & $438 * 451 * 301 \mathrm{~mm}, 2935 \mathrm{~g}$ (total \\
weight & weight) \\
Flight mode & Dual, based on wireless control \\
Endurance & $18 \mathrm{~min}$ \\
Camera & Digital Gimbal, X3 (focal length \\
configurations & 20mm) \\
Ground Control & Telemetry for real time flight \\
Station & control, path tracking on video \\
\hline
\end{tabular}

Table 1. Some key specifications of the UAV system

\subsection{Flight Planning}

Before flight plan an operator needs to identify the coverage of the study area in sequence to design flight plan. The flight plan requires an approximate coordinates as an input to design the flight route. This study used aerial photogrammetric method as a primary method in data acquisition. Therefore, user needs to determine the percentage of overlap area and side lap area between the pair of the images in the flight plan. Site Preparation involves developing a layout for ground control points, checking the accuracy of the camera image used for flight planning, ensuring no obstacles are in the path of the $\mathrm{UAV}$, and evaluating environmental conditions to determine if a flight can be safely undertaken. Surveys were conducted in 13, January and 6, April 2016.

\subsection{GCP Surveying}

For ground survey work, the imagery needs to be geo-corrected. A network of Ground Control Points (GCP) accurately surveyed with a RTK-VRS, which is provides instant access to real-time kinematic (RTK) corrections utilizing a network of permanent (fixed) continuously operating reference stations, or identified in precise map source are necessary for accurate location. GCP are usually markers placed in a regular network 
on the ground and may comprise a post plus a cross (and number) visible on the aerial photograph. Each aerial image acquired has to be corrected to a true position on the Earth's surface with the aid of digital image processing software. The creation of each GCP should follow certain guidelines. Most importantly, the GCP needs to be identifiable in the images taken by the UAV. This requires each GCP to be large enough to be seen by the imaging equipment.

The RTK-VRS survey, performed by a dual frequency GSX2 (Sokkia) for the mentioned dataset (GCPs), resulted in RMS values of less than $0.02 \mathrm{~m}$ and $0.03 \mathrm{~m}$ for horizontal and vertical accuracies respectively, for $98 \%$ of the sampled points. Horizontal coordinates were referenced to UTM zone $52 \mathrm{~N}$, while the vertical values were referred to the mean sea level using the geoid model KNGeoid13 provided by the National Geographic Information Institute (NGII). For this study, it was determined that an appropriate number of GCPs for the site would be between 34 and 37 (Figure 3).

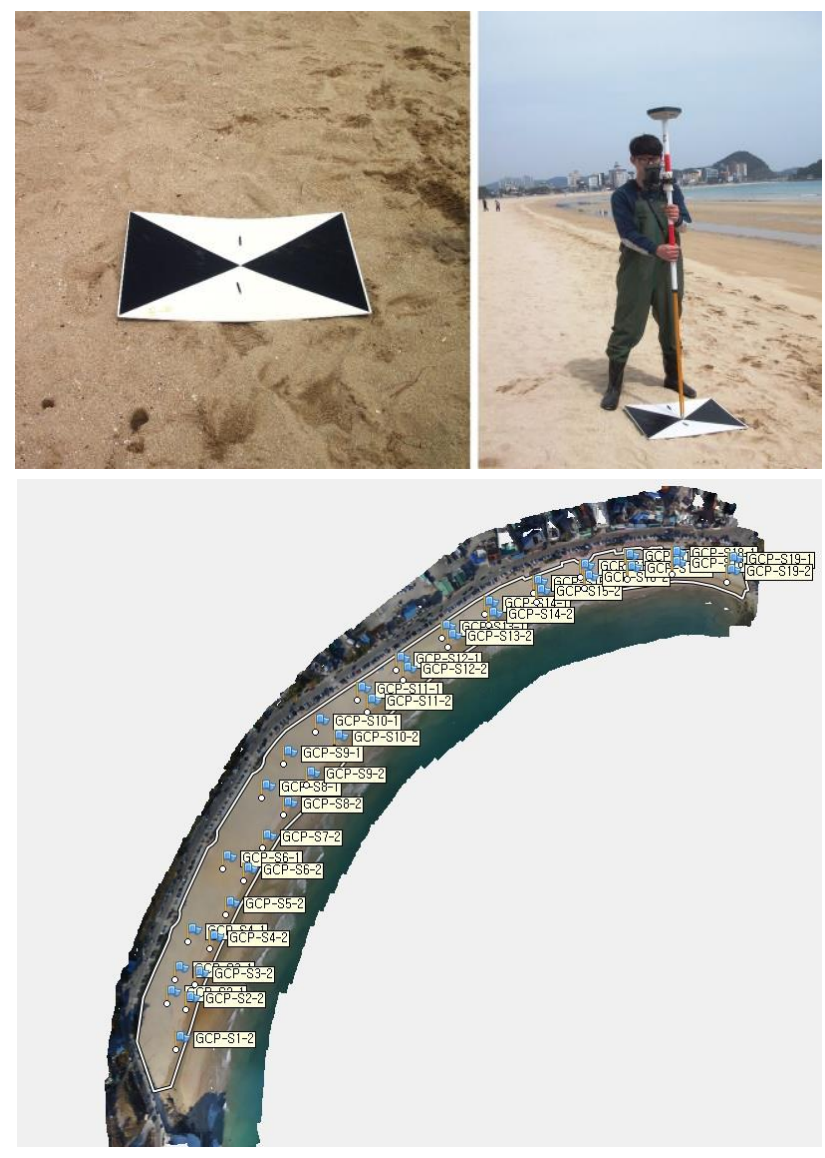

Figure 3. Set of GCP locations.

\subsection{Photogrammetric Processing and Software}

The processing of original image collection was performed by the software package called PhotoScan, available at a moderate cost for research institutions from the Russian manufacturer AgiSoft LLC. The SfM algorithm implemented by PhotoScan was used in this work to generate the dense DSM of the study area to be successively validated with the GNSS GCPs and compared with the DSM produced by the more familiar TLS survey technique. The reconstruction of ground surface and objects by PhotoScan is a three-step process. For a good reconstruction, at least two photographs representing a single point must be available.

In the first step the alignment of the acquired images was performed. The SfM algorithm comes into play by the detection of image feature points and reconstruction of their movement along the sequence of images. In the second step a pixel-based dense stereo reconstruction was performed starting from the aligned dataset. After this step, fine topographic details available on the original images could be meshed. In the third step a texturing was applied to the mesh. Despite the good level of automation of the whole procedure, the computation could be time consuming or limited by the amount of Ram available. The point cloud was framed in the UTM coordinates system. In the last step, DEM and orthophoto (orthomosaic), which were geo-referenced to the WGS84 spheroid and datum, respectively.

\section{RESULTS}

\subsection{Photogrammetric Processing}

The UAV performed 2 times (13 January, 6 April 2016) of flights, gathering approximately 3 gigabytes of aerial imagery. The initial processing of 180 images (ground resolution of $0.003 \mathrm{~m} /$ pixel at $50 \mathrm{~m}$ flying height) and the final processing of 1036 images (ground resolution of $0.004 \mathrm{~m} /$ pixel at 30, 50, 70 m flying height) acquired by the UAV system (Figure 4).
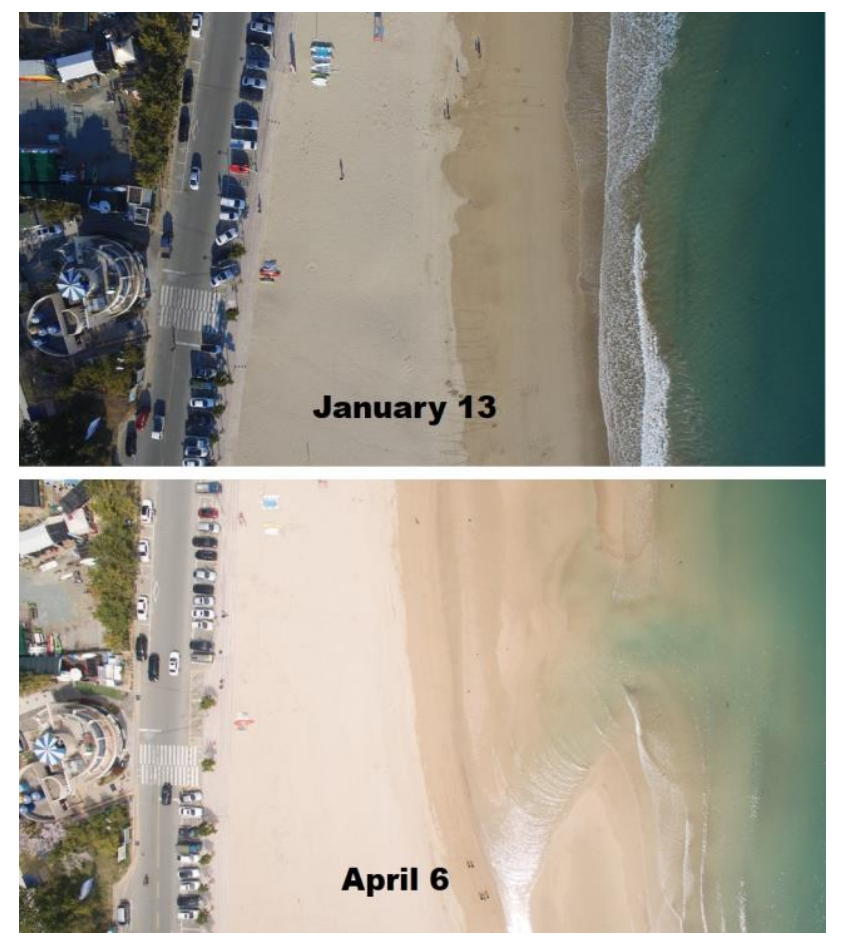

Figure 4. Aerial images acquired by UAV on Songjung beach.

By Knowledge of the GCPs coordinates, after processing by the SfM approach, bundle adjustment was performed to register the model in the UTM reference system camera locations and attitude were considered as unknowns.

Successively, for further investigations on the absolute accuracy of 3D surface from the point cloud, a linear interpolator was used to produce a DSM. The pixel spacing was selected on the basis of the average distance between points of the dense cloud. This resulted in a $2.93 \mathrm{~cm}$ resolution 
DSM leading to a densification of points for areas where the density of points was major.

\subsection{Analysis of topographic and morphologic changes}

The basic method for topographic change identification using DEMs is differencing the $\mathrm{Z}$ coordinates of the 6 April to the 13 January (Figure 5). The volume change at each cell location can then be computed. A positive, negative, or zero volumetric value $\left(\mathrm{m}^{3}\right)$ at $\mathrm{c}$ cell represents the amount of deposition, erosion, or no change. The morphological change of topography over the entire study area can be obtained through summing the positive, negative volumetric values in each cell.

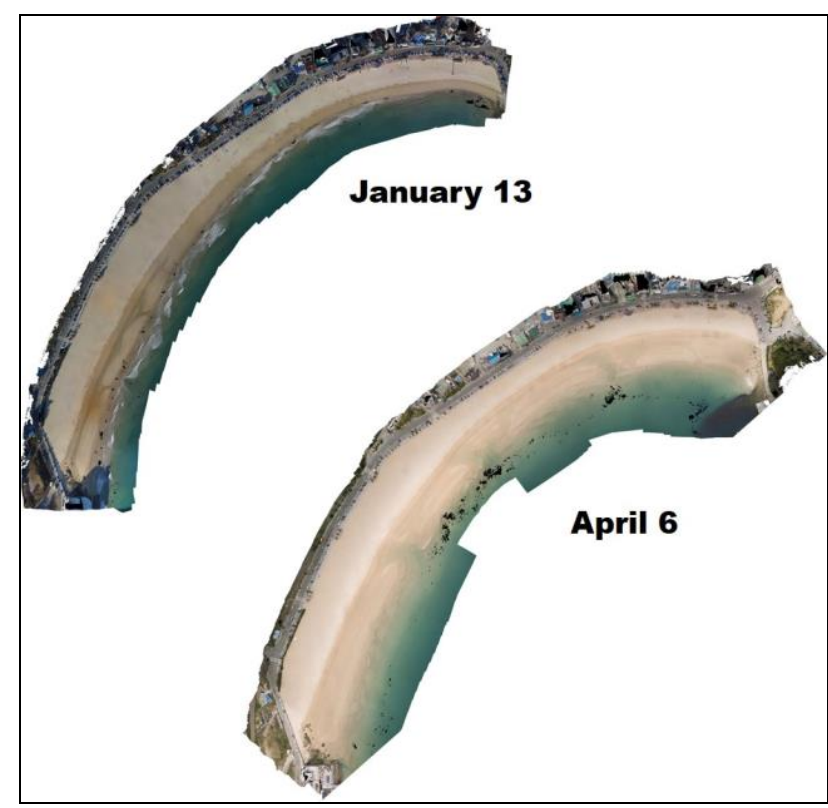

Figure 5. Comparison with orthophotos in study area.

Beach profiles were surveyed at 19 locations in the study area (Figure 4) by means of RTK-VRS. Beach volume change for DEMs was calculated using the Regional Morphology Analysis Package (Rmap). Rmap is part of the Coastal Engineering Design and Analysis System (CEDAS) software. This software provides tools for the manipulation, analysis, visualization, and archiving of shoreline position and beach profile data.

\section{DISCUSSION}

Current methods of coastal surveying include Terrestrial Laser Scanning, airborne-Light Detection and Ranging (LiDAR) scans, and Real Time Kinematic Global Positioning Surveys. These methods are time consuming and resource intensive. Unmanned Aerial Vehicles are gaining popularity amongst both the public and researchers, and could offer a cheaper alternative to airborne and LiDAR. UAVs can also track coastal erosion along stretches of beach on a more frequent basis. Using traditional satellite sensors and manned aerial systems, however, can be challenging due to issues related to cloud cover, mobilization expenses and resolution. Rapid advances in unmanned aerial vehicle (UAV) technology now allows for cost-effective collection of aerial imagery and topography at centimetre resolution suitable for assessing change in coastal ecosystems.
The EUROSION study (2004) recommends to deal with coastal erosion by restoring the overall sediment balance on the scale of coastal cells, which are defined as coastal compartments containing the complete cycle of erosion, deposition, sediment sources and sinks and the transport paths involved. UAV survey is very useful for measuring coastal cell

The current method for georeferencing the images uses GCPs. In a coastal area, especially beaches, there are very few invariant natural GCPs. So for each UAV flight it is required to do DGPS surveying on different GCPs, which increases the costs and limits the interest of using an UAV. Direct georeferencing (Schwarz et al., 1993, Shukla and Smith, 2000) might represent a valid alternative to this problem. Direct georeferencing most often implies the use of a very high quality GNSS receiver and an INS, which implies load and cost increases. Another solution is to use only the positions of camera center for each shot without attitude measurements, with GNSS only measurements (Turner et al., 2013).

One reason topography is measured in the Songjung Beach is to monitor the volumetric changes of the sediment deposits as a function of river regime. To determine the effect that the observed UAV elevation accuracies have on estimating the volume of sediment, we selected sites with the densest ground surveys within beach.

\section{CONCLUIONS}

The study of beach volume change at Songjung beach demonstrate that the integration of UAV techniques and photogrammetric software and analysis tools can provide new insights into the short term evolution of coastal landscapes by quantifying topographic change at high levels of spatial and temporal detail. Such results can provide important information for beach management decision making as well as methodologies and tools that can be applied to other coastal regions.

\section{ACKNOWLEDGEMENTS (OPTIONAL)}

This research was supported by Basic Science Research Program through the National Research Foundation of Korea (NRF) funded by the Ministry of Education. (NRF2015R1D1A1A01060446)

\section{REFERENCES}

Anon. 1993. Coastal defence and the environment: a guide to good practice. MAFF, London.

Marzolff, I., Poesen, J., 2009. The Potential of 3D gully monitoring with GIS using resolution aerial photography and a digital photogrammetry system. Geomorphology, 111 (1-2), pp. 48-60.

EUROSION, 2004. A guide to coastal erosion management practices in Europe. Contact B4-3301/2001/329175/MAR/B3. Prepared by Rijkswaterstaat/RITZ, The Haque, The Netherlands.

Schwarz, K. Chapman, M., Cannon, M., and Gong, P., 1993. An Integrated INS/GPS Approach to the Georeferencing of Remotely Sensed Data. Photogrammetric Engineering and Remote Sensing, 59(11). 667-1674 
Shukla. R.K. and Smith, M. J., 2000. Geo-referencing casi imagery using direct measurement of position and attitude. In: International Archives of Photogrammetry and Remote Sensing, XXXIII (Part B2), 502-509.

Schwarz, K. Chapman, M., Cannon, M., and Gong, P., 1993. An Integrated INS/GPS Approach to the Georeferencing of Remotely Sensed Data. Photogrammetric Engineering and Remote Sensing, 59(11). 667-1674

Turner, D., Lucieer, A., and Wallac, L., 2013. Direct Georeferencing of Ultrahigh- Resolution UAV Imagery. IEEE Transactions on Geoscience and Remote Sensing, 52(5), 27382745 . 